\title{
Interchain Charge Transfer States Mediate Triplet Formation in Purified Conjugated Polymer Aggregates
}

Alan K. Thomas, Hunter A. Brown, Benjamin D. Datko, Jose A. Garcia-Galvez, John K. Grey*

\author{
Department of Chemistry and Chemical Biology, \\ University of New Mexico, \\ Albuquerque, NM 87131 USA
}

\section{Supporting Information}

Absorption spectra were measured from aged nanofibers as well as aged fibers that were "reconstituted" via sonication or dilution (see Figure S1). These spectra show that although Haggregate character develops as a function of nanofiber aging in solvent solutions, this character can be reverted to the pristine J-aggregate absorption via sonication or dilution.

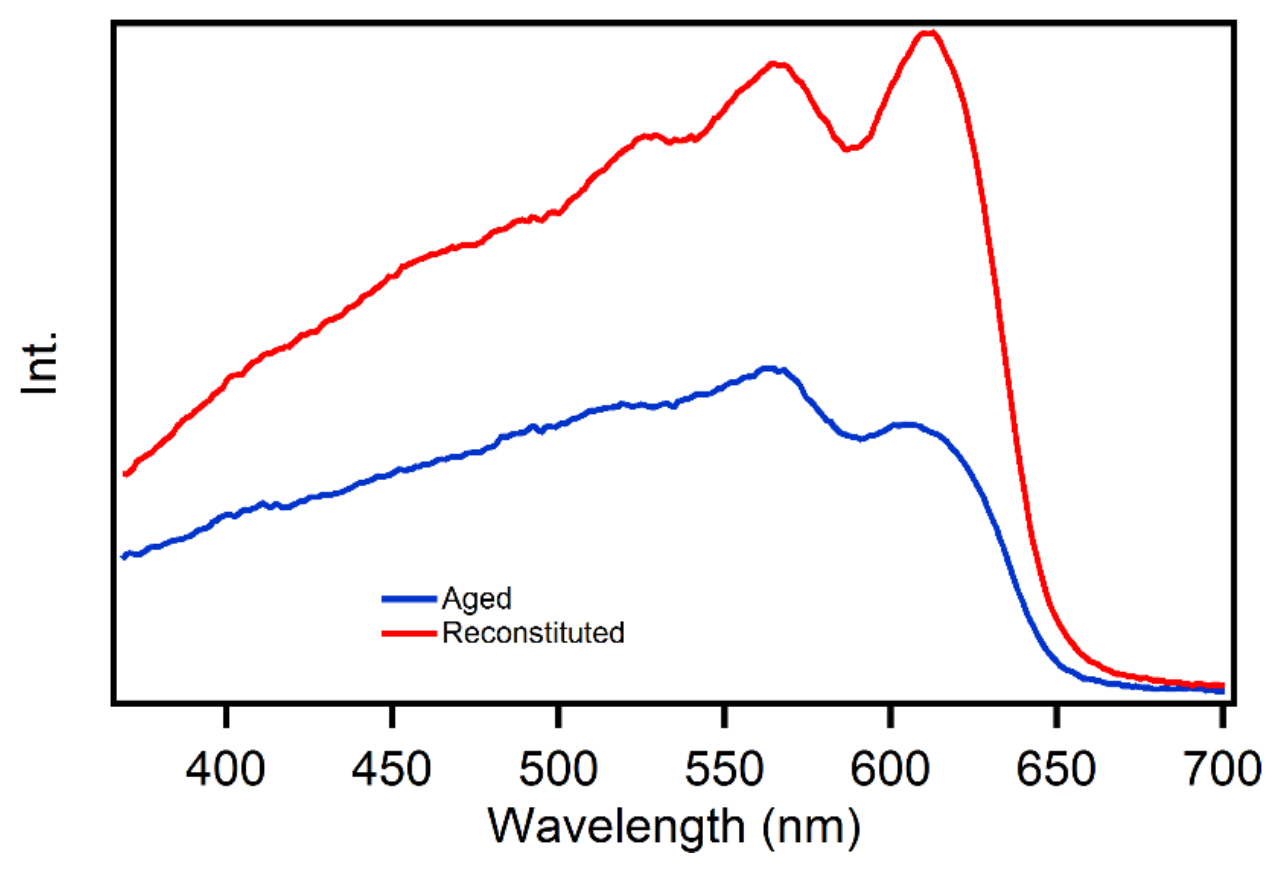

Figure S1. UV-Vis absorption spectra of P3HT nanofibers. 
Representative PL spectra from individual fibers are shown in Figure S2 (below). As quenching increases in an individual fiber there is a decrease in PL intensity coupled with a decrease in the $0-0 / 0-1$ peak ratio. This is due to the fact that unquenched PL probably originates from regions of the nanofiber which are devoid of chain-chain contacts that support charge transfer states (see main text).

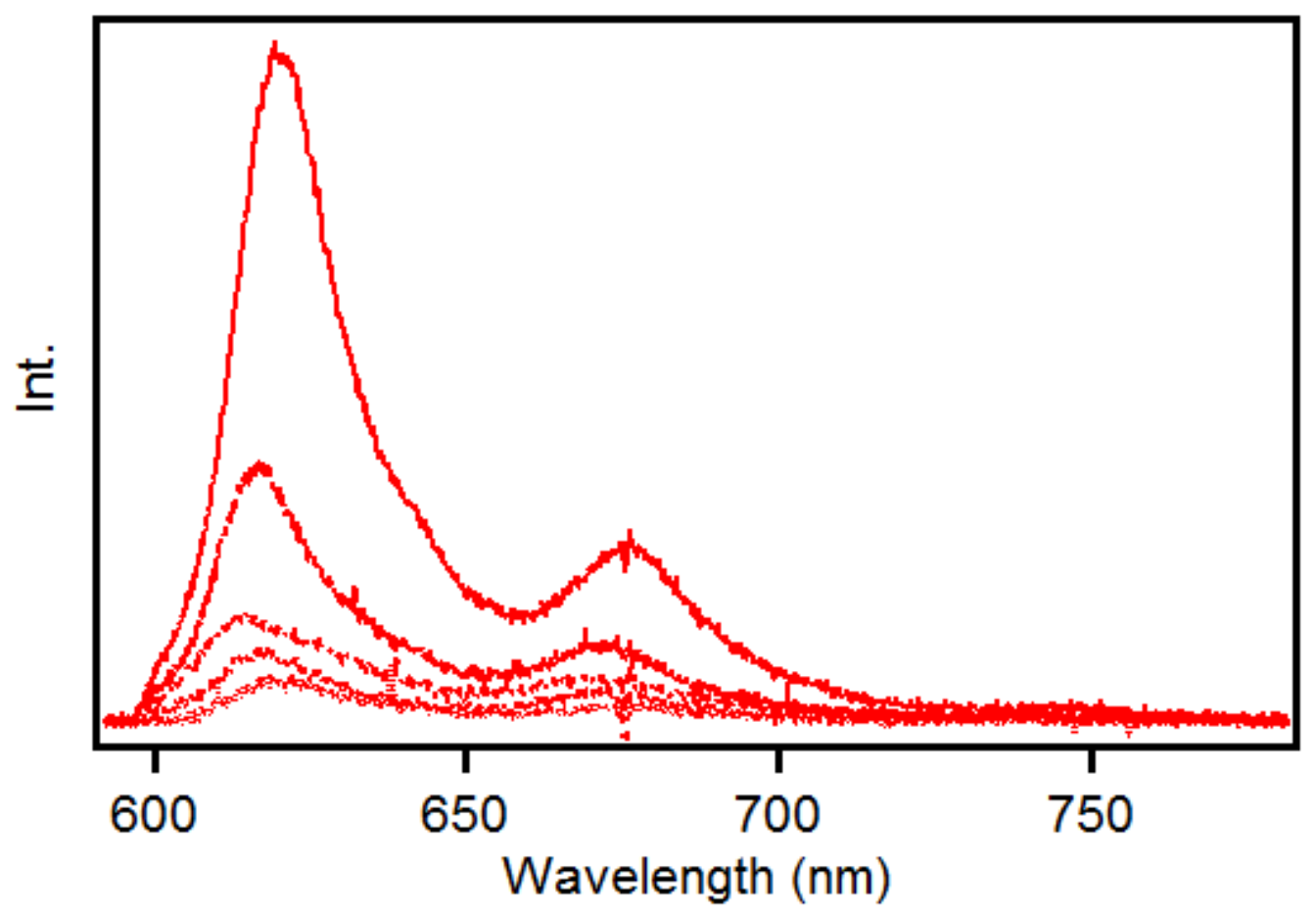

Figure S2. PL spectra of individual P3HT nanofibers. 


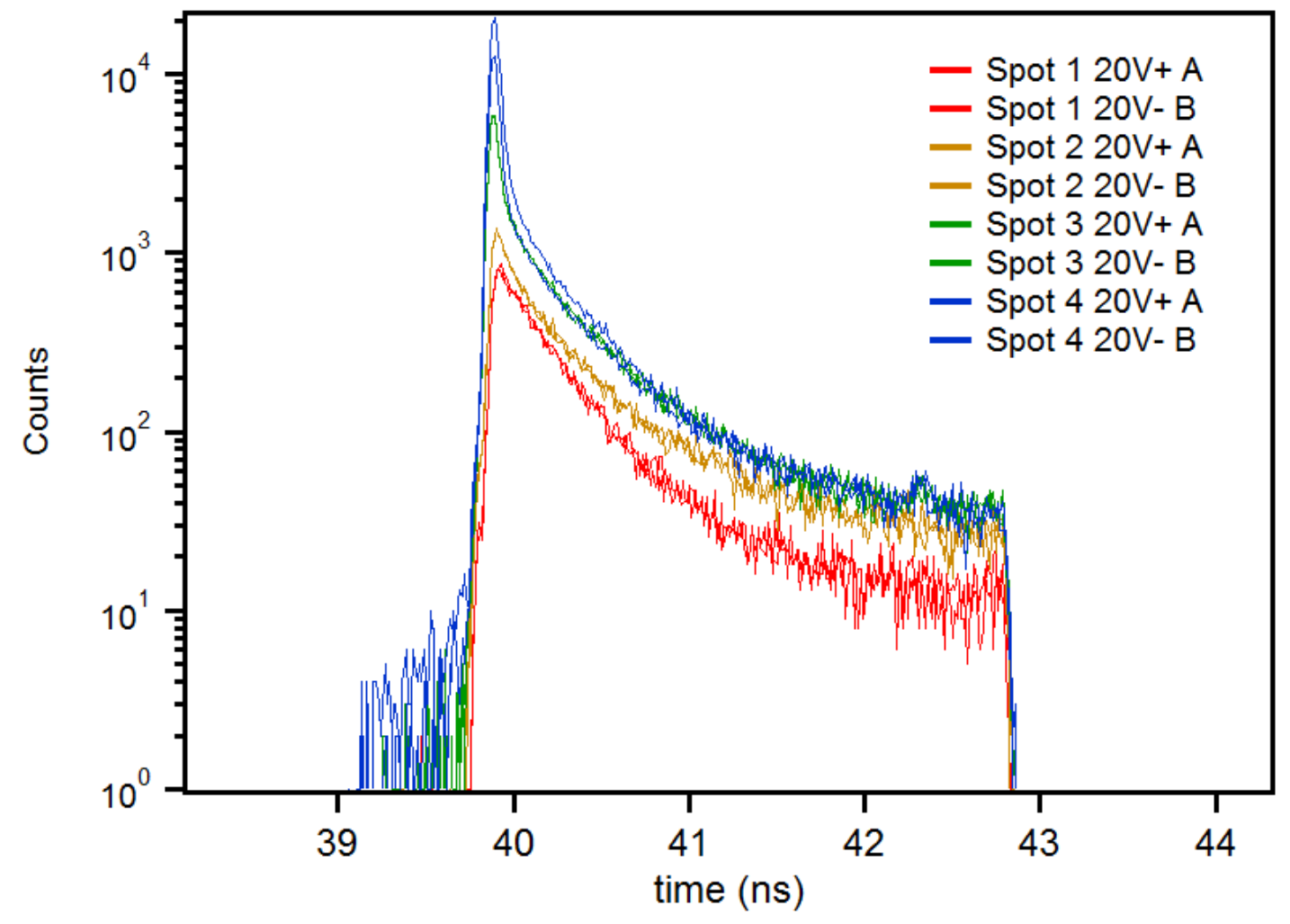

Figure S3. Representative TCSPC spectra of individual P3HT nanofibers with positive and negative bias.

The data presented in Figure S3 shows the bias dependent decays from a number of individual fibers. This data shows that the direction of the bias has little effect on the overall decay transient lineshape, which is consistent with randomly oriented nanofibers in the support matrix. Regardless of the absolute intensity of the PL from an individual fiber, the decay fits also returned similar lifetimes from each individual fiber under either bias direction. 


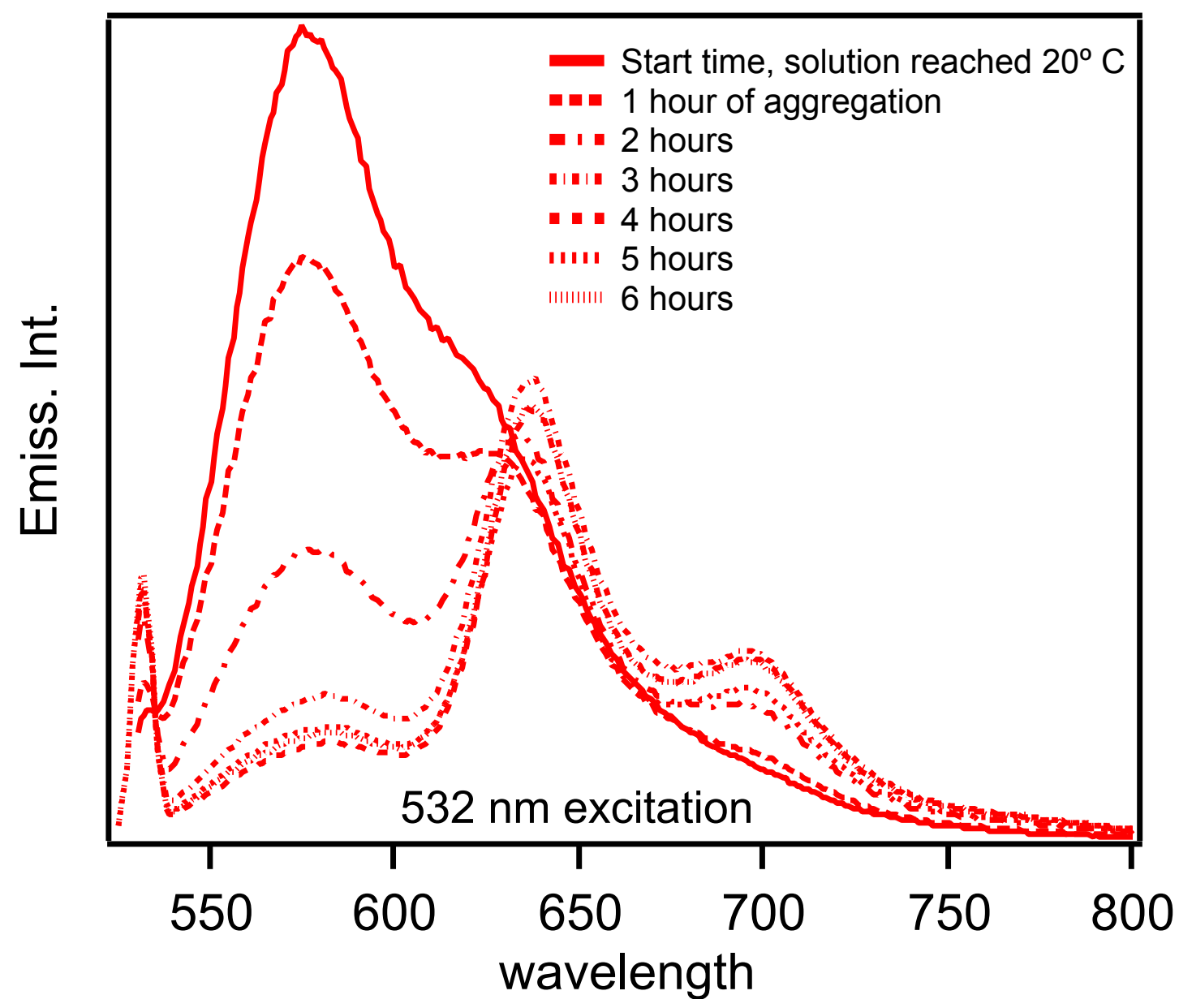

Figure S4. Time evolution of PL spectrum from nanofibers during formation.

Figure S4 shows emission spectra taken with $532 \mathrm{~nm}$ excitation from solutions during nanofiber formation. The peak located $\sim 575 \mathrm{~nm}$ is the emission of the amorphous material, which decreases with aggregation time and can be separated by purification (i.e., centrifugation). The peak near $\sim 630 \mathrm{~nm}$ is the $0-0$ emission peak which grows in as a function of aggregation time. 


\section{Multi-state photodynamic model of triplet-induced PL quenching}

Triplet formation in P3HT J-aggregates is proposed to proceed via recombination of charge transfer states, which takes place on timescales $>10$ ns. Similar to previous single molecule spectroscopic investigations of PPV-type and push-pull systems, ${ }^{1,2}$ the presence of triplets was confirmed by monitoring the quenching of PL emission intensity over time. Quenching dynamics were explained using a coupled multi-chromophore model that considers the amounts of singlet and triplet excited states on two interacting sites (chromophores). The following outlines the framework of the model.

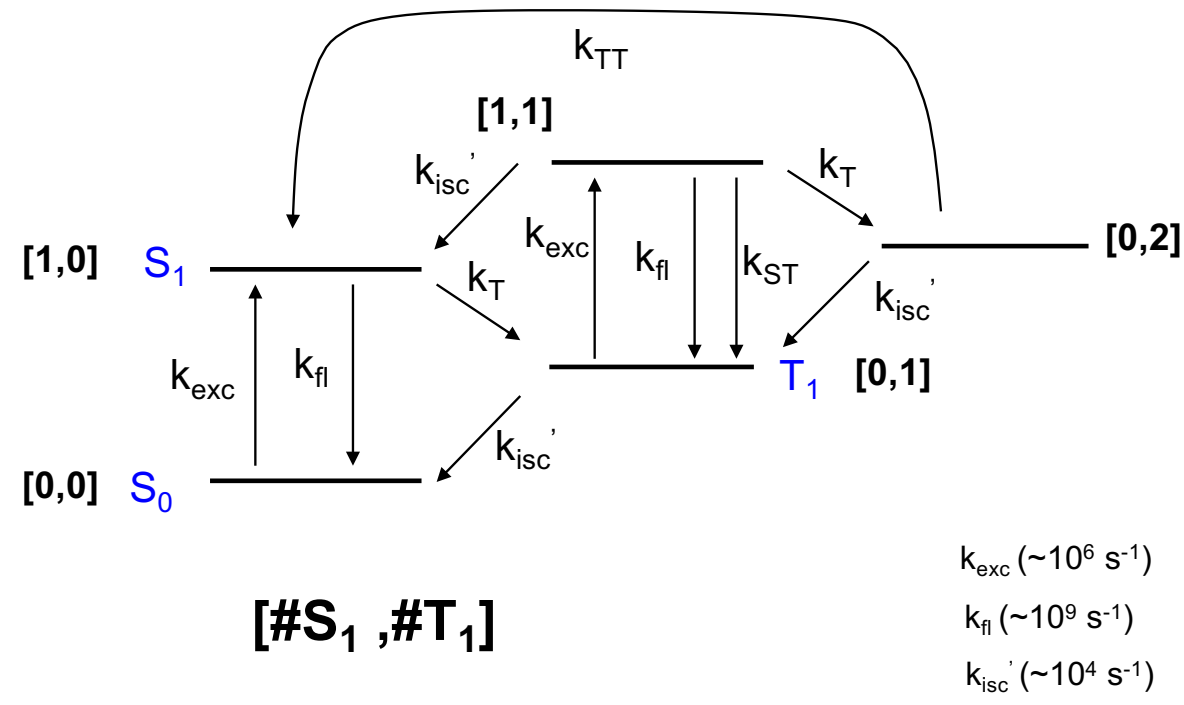

2 site, coupled chromophore dynamic model

$\underline{\text { Rate equations }}$

$\frac{d[0,0]}{d t}=-k_{\text {exc }}[0,0]+k_{P L}[1,0]+k_{i s c}^{\prime}[0,1]$

$\frac{d[1,0]}{d t}=k_{\text {exc }}[0,0]-\left(k_{P L}+k_{T}\right)[1,0]+k_{i s c}^{\prime}[1,1]$

$\frac{d[0,1]}{d t}=k_{T}[1,0]-\left(k_{\text {exc }}+k_{i s c}^{\prime}\right)[0,1]+\left(k_{P L}+k_{S T}\right)[1,1]+k_{i s c}^{\prime}[0,2]$ 


$$
\begin{aligned}
& \frac{d[1,1]}{d t}=k_{\text {exc }}[0,1]-\left(k_{P L}+k_{S T}+k_{i s c}^{\prime}+k_{T}\right)[1,1] \\
& \frac{d[0,2]}{d t}=k_{T}[1,1]-\left(k_{T T}+k_{i s c}^{\prime}\right)[0,2]
\end{aligned}
$$

\section{Quantum Yields}

$\Phi_{f l}^{[1,0]}=\frac{k_{P L}}{\left(k_{P L}+k_{T}\right)}$

$\Phi_{T}^{[1,0]}=\frac{k_{T}}{\left(k_{P L}+k_{T}\right)}$

$\Phi_{f l}^{[1,1]}=\frac{k_{P L}}{\left(k_{P L}+k_{S T}+k_{i s c}^{\prime}+k_{T}\right)}$

$\Phi_{T}^{[1,1]}=\frac{k_{T}}{\left(k_{P L}+k_{S T}+k_{i s c}^{\prime}+k_{T}\right)}$

$\Phi_{T^{\prime}}^{[1,1]}=\frac{k_{i s c}^{\prime}}{\left(k_{P L}+k_{S T}+k_{i s c}^{\prime}+k_{T}\right)}$

$\Phi_{T T}^{[0,2]}=\frac{k_{T T}}{\left(k_{T T}+k_{i s c}^{\prime}\right)}$

$\left(k_{P L} \sim k_{r}+k_{n r}\right)$

Earlier studies of triplet PL quenching in single polymer molecules assumed a second-order perturbative mechanism for the triplet formation rate constant. ${ }^{1}$ As stated in the main text, this mechanism is probably not operative in $\mathrm{P} 3 \mathrm{HT}$ J-aggregates due to unfavorable symmetry requirements for efficient singlet-triplet mixing via the spin-orbit operator. Regardless, decay of triplets back to the ground state $([0,0])$ should proceed via reverse intersystem crossing described by $k_{i s c}^{\prime}$ since no phosphorescence is observed. In P3HT aggregates, triplet lifetimes were estimated to be $\sim 10 \mu$ s in agreement with previous reports. $^{3}$ Estimates of the second order triplet-triplet annihilation rate constants are much less straightforward to determine as well as relative populations of the $[1,1]$ and $[0,2]$ states.

Yu et al. were able to simplify this model by assuming that $[1,0],[1,1]$, and $[0,2]$ are sufficiently small and do not change with time thus allowing the steady-state approximation to be applied. ${ }^{1}$ Additionally, if triplet formation yields are small, $\Phi_{T T}^{[0,2]}$ is near unity and $k_{T}>>k_{i s c}^{\prime}$ eq. 3 becomes,

$\frac{d[0,1]}{d t}=k_{\text {exc }} \Phi_{T}^{[1,0]}[0,0]+\left(k_{i s c}^{\prime}+k_{\text {exc }} \Phi_{T}^{[1,1]}\right)[0,1]$ 
which is identical to the expression found in Refs. 1 and 2. Here, the system spends the most time in the $[0,0]$ and $[0,1]$ configurations and the model now reduces to an effective two-level system,

$[0,0] \leftrightharpoons[0,1]$

with forward $\left(k_{f}\right)$ and reverse $\left(k_{b}\right)$ rate constants of,

$k_{f}=k_{\text {exc }} \Phi_{T}^{[1,0]}$

$k_{b}=k_{i s c}^{\prime}+k_{\text {exc }} \Phi_{T}^{[1,1]}$

Application of the model involves solving the above differential equations for the probability of forming one triplet (i.e., $[0,1])$,

$\frac{d P_{1}}{d t}=k_{f} P_{1}-k_{b}\left(1-P_{1}\right)$

The time-dependent fluorescence intensity modulation is given by,

$I(t) \propto I_{\text {exc }}(t)\left[\left(1-P_{1}(t)\right)+\frac{\tau_{f l}^{\prime}}{\tau_{f l}} P_{1}(t)\right.$

where $\tau_{P L}^{\prime}$ and $\tau_{P L}$ are the PL lifetimes of the $[1,1]$ and $[1,0]$ states. ${ }^{1,2} I_{\text {exc }}(t)$ is the excitation intensity, which corresponds to the square shape pulses used to excite the fluorescence described in the main text and Refs. 1 and 2. We show how this simple model predicts buildup of triplet populations and induces PL quenching in single P3HT J-aggregates (Figure 2, main text). Although this model can be used in a semi-quantitative fashion, reliable estimates of the singlet-triplet quenching and triplet annihilation rate constants, $k_{T T}$ and $k_{S T}$, in addition to [1,1] and [0,2] populations are difficult to obtain.

In particular, application of this two-level model requires that triplet formation yields are small in order to reduce to an effective two-level system. For polythiophenes, it is unlikely that $[0,2]$ populations are small enough to invoke the steady state approximation for eq. 5. In this regime, eqs. 1-5 are described by the following simultaneous and inhomogeneous rate equations,

$$
\begin{aligned}
& \frac{d[0,0]}{d t}=\left(1-\Phi_{T}^{[1,0]}\right) k_{T T}-\left\{k_{\text {exc }}+\left(1-\Phi_{T}^{[1,0]}\right)\left(k_{T T}-k_{\text {exc }}\right)\right\}[0,0]+\left(1-\Phi_{T}^{[1,0]}\right)\left(k_{\text {exc }} \Phi_{T^{\prime}}^{[1,1]}-\right. \\
& \left.k_{T T}\right)[0,1]
\end{aligned}
$$




$$
\begin{aligned}
\frac{d[0,1]}{d t}=\left(k_{i s c}^{\prime}\right. & \left.+k_{T T} \Phi_{T}^{[1,0]}\right)-\left\{k_{i s c}^{\prime}+\left(k_{T T}-k_{\text {exc }}\right)[0,0]\right. \\
& -\left\{2 k_{i s c}^{\prime}+k_{e x c}+\left(k_{T T}-k_{e x c} \Phi_{T^{\prime}}^{[1,1]}\right)-\frac{k_{e x c}\left(k_{P L}+k_{S T}\right)}{\left(k_{P L}+k_{S T}+k_{i s c}^{\prime}+k_{T}\right)}\right\}
\end{aligned}
$$

Eqs. 18 and 19 are solvable by setting $[0,0]=1$ at $\mathrm{t}=0$, however, solutions are quite complicated and we do not attempt to fit experimental quenching data at this time. In order to further assess the validity of this

model, we are currently exploring heavy atom analogs of P3HT which are expected to reveal changes in triplet populations and quenching efficiency but this analysis will be published at a later time.

\section{References}

1. Yu, J.; Lammi, R.; Gesquiere, A. J..; Barbara, P. F., Singlet-Triplet and Triplet-Triplet Interactions in Conjugated Polymer Single Molecules. Journal of Physical Chemistry B 2005, $109,10025-10034$.

2. Gesquiere, A. J.; Lee, Y. J.; Yu, J.; Barbara, P. F., Single Molecule Modulation Spectroscopy of Conjugated Polymers. Journal of Physical Chemistry B 2005, 109, 1236612371.

3. Thomas, A.K.; Garcia, J.A.; Ulibarri-Sanchez, J.; Gao, J.; Grey, J.K., High intra-chain order promotes triplet formation from recombination of long-lived polarons in poly(3hexylthiophene) J-aggregate nanofibers. ACS Nano, 2014, 8, 10559-10568. 